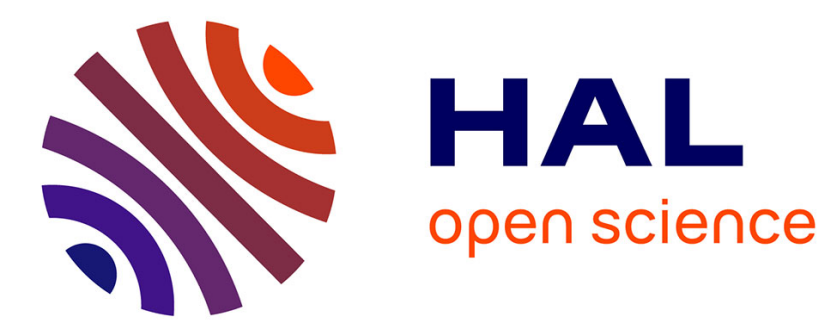

\title{
Water Transport in Bio-based Porous Materials A Model of Local Kinetics of Sorption-Application to Three Hemp Concretes
}

N. Reuge, S. Moissette, Marjorie Bart, F. Collet, Christophe Lanos

\section{- To cite this version:}

N. Reuge, S. Moissette, Marjorie Bart, F. Collet, Christophe Lanos. Water Transport in Bio-based Porous Materials A Model of Local Kinetics of Sorption-Application to Three Hemp Concretes. Transport in Porous Media, 2019, 128 (2), pp.821-836. 10.1007/s11242-019-01272-4 . hal-02122197

HAL Id: hal-02122197

https://hal-univ-rennes1.archives-ouvertes.fr/hal-02122197

Submitted on 18 Jul 2019

HAL is a multi-disciplinary open access archive for the deposit and dissemination of scientific research documents, whether they are published or not. The documents may come from teaching and research institutions in France or abroad, or from public or private research centers.
L'archive ouverte pluridisciplinaire HAL, est destinée au dépôt et à la diffusion de documents scientifiques de niveau recherche, publiés ou non, émanant des établissements d'enseignement et de recherche français ou étrangers, des laboratoires publics ou privés. 

three hemp concretes

\author{
N. Reuge ${ }^{1, *}$, S. Moissette ${ }^{1}$, M. Bart ${ }^{1}$, F. Collet ${ }^{1}$, C. Lanos $^{1}$ \\ ${ }^{1}$ Laboratoire de Génie Civil et Génie Mécanique, Axe Ecomatériaux pour la construction, Université de \\ Rennes, 3 rue du Clos Courtel, BP 90422, 35704 Rennes, France
}

*Corresponding author, e-mail: reuge@free.fr

\begin{abstract}
The classic models describing the hygric mass transfers inside porous materials seem unsuitable in the case of bio-based materials. They are based on the assumption of instantaneous local equilibrium between relative humidity and water content (Künzel 1995). These two parameters evolve according to the diffusive fluxes following the sorption isotherms. This study shows that it leads to predict much shorter times of stabilization than those experimentally obtained. A new approach is presented here, it frees from the local instantaneous equilibrium introducing a local kinetics to describe the transformation of water from vapor state to liquid state and vice versa. The local kinetics of sorption is coupled with the well-known hysteresis phenomenon. It is adjusted from bibliographic data (Collet et al. 2013) giving mass evolution of three hemp concrete under adsorption / desorption conditions. 1D cylindrical simulations allows an excellent fitting on the experiments. Finally, a semiempirical model is proposed, allowing to determine the kinetics parameters more easily.
\end{abstract}

Keywords: bio-based porous materials, hemp concrete, local kinetics, sorption, hygric transfer, modeling.

\title{
Acknowledgments
}

This work has been performed and funded in the framework of the European project ISOBIO (http://isobioproject.com) within the scope the of the research and innovation program Horizon 2020 (agreement No. 636835).

\section{Introduction}

Water sorption in porous media involves complex and coupled phenomena such as vapor / liquid water mass transport by Fickian diffusion and equilibrium isotherms of adsorption / desorption also called "water storage functions" associated with hysteretic phenomena. The diffusion coefficients can be determined in steady state exchange by permeability measurements (i.e. "wet cup method" (Kumaran 1998)). The equilibrium isotherms of sorption can be determined by gravimetric methods (Iglesias and Chirife 1982). Actually, the equilibrium water contents evolve as a function of the relative humidities between the main adsorption and desorption equilibrium isotherms depending on the material hygric history, this complex phenomenon is rather well understood and can be described by hysteretic models (Carmeliet et al. 2005; Huang et al., 2005). But despite the knowledge and the reliability of these hygric models, it seems that inside some porous materials, such models largely 
underestimate the time required for the water content to reach the equilibrium: it has been established for bio-based materials (Nyman et al. 2006; Frandsen et al. 2007; Eitelberger and Svensson 2011; Alexandersson et al. 2016) as well as for more traditional material such as cement compounds (Johannesson and Nyman 2010; Zeng et al. 2015). These considerations take on their full meaning in the cases of hygric transfers with permanent fluctuations such as the hygric transfers occurring in materials of building envelops, in soils or in food materials.

In this study, we focus on the case of hygric transfers in bio-based building materials: the hemp concretes.

Classic simulation tools of hygrothermal transfers in building materials are based on the assumption that for a given local relative humidity $(\varphi)$, the associated equilibrium local water content $(w)$ is reached instantaneously. In this study, three hemp concretes are studied and it is demonstrated that such an assumption leads to serious inconsistencies.

Thus, as reported previously in literature (Nyman et al. 2006; Frandsen et al. 2007; Eitelberger and Svensson 2011; Alexandersson et al. 2016; Maraqa 2016), a local kinetics of sorption may exist (from water vapor to liquid water and inversely) which is slow compared to the diffusive fluxes. Obviously, this invalidate the aforementioned assumption. Several expressions for the local kinetics of sorption can be found in the aforementioned references. In this study, an expression is proposed and validated for the three considered hemp concretes whatever the hygric operating conditions (relative humidities, adsorption or desorption phases).

The first part of this study is a summary of the hygric characterization performed by (Collet et al. 2013) of three selected hemp concretes. Then, the theoretical background necessary to model the water sorption is presented. Expressions of the local kinetics of sorption found in literature are discussed and a new expression is proposed. The associated parameters are a kinetics constant and a driving force.

A preliminary study based on the simulations of hygric transfers during sorption tests invalidates the calculations performed with traditional models using the assumption of instantaneous $\varphi / \mathrm{w}$ equilibrium. These results lead to introduce an adequate expression for the local kinetics of sorption. In the following part, the new transfer model is validated for the three studied hemp concretes against the experiments performed by (Collet et al. 2013) and the results are discussed. In the last part, a semiempirical analytical model is proposed, allowing to estimate straightly the kinetic parameters.

\section{Experimental characterizations}

\subsection{Materials properties}

The three studied hemp concretes are made of hemp shiv mixed with mineral binders. Their properties had been characterized in (Collet et al. 2013). The first one is a precast hemp concrete (PHC) also known as EASY- $\mathrm{R}^{\mathrm{TM}}$. The components of its mineral matrix are $\mathrm{CaO}(72 \%)$ and lime (28\%) with a hemp/binder mass ratio of 0.65 . Its dry bulk density is of $450 \mathrm{~kg} \cdot \mathrm{m}^{-3}$ and its open porosity is of 0.68 . The second one is a sprayed hemp concrete $(\mathrm{SHC})$ with a lime based binder (Tradical $70^{\mathrm{TM}}$ ), a hemp/binder mass ratio of 0.5 , a dry bulk density is of $425 \mathrm{~kg} \cdot \mathrm{m}^{-3}$ and an open porosity of 0.66 . The third one is a molded hemp concrete (MHC) with the same lime based binder as SHC, a hemp/binder mass ratio of 0.5 , a dry bulk density is of $425 \mathrm{~kg} \cdot \mathrm{m}^{-3}$ and an open porosity of 0.77 . Therefore, the properties of these hemp concretes are different due to different mineral matrixes components and/or different hemp/binder mass ratio and/or different manufacturing processes. 
Extensive permeability measurements had been performed by (Collet et al. 2013, Chamoin 2014) by the wet cup method. They led to water vapor resistance factor $\mu$ of $4 \pm 30 \%$ for the three studied materials in the range of 0 to $50 \%$ ambient relative humidity $(R H)$. For $R H$ higher than $60 \%$, the permeabilities tend to increase: this is due to the initiation of liquid transport which becomes really significant for $R H$ higher than $80 \%$. As done by (Ouzemiane 2013) and more usually done in the relevant area (Künzel 1995), $\mu$ will be still considered as constant and the increase of permeability only due to the increasing contribution of liquid transport. However, we have no proof that these permeability increases is not accompanied by a change of the water vapor diffusion resistance factor, the water vapor transport by diffusion probably decreases significantly at very high $R H(>80 \%)$ due to waterlogging of the macro-porous network by free water, but this is more than compensated by the liquid water transport since the global permeabilities increase a lot.

\subsection{Global kinetics of sorption}

\subsubsection{Operating conditions}

The adsorption/desorption behaviors of these hemp concretes had been measured by (Collet et al. 2013) using samples of $5 \mathrm{~cm}$ characteristic diameter and sealed on their ends. It is important to note that such an important size is required for the samples of these composite materials to be representative. In order to ensure the repeatability of the results, the measurements had been performed on six samples for each material.

The sorption curves were measured according to a discontinuous method: the time dependent water content was determined at successive stages of increasing (and then decreasing) relative humidity. The samples were placed in a climatic chamber (Vötsch VC4060) with temperature and relative humidity regulations. They were weighed two to three times a week. The measurements were done at a temperature of $23 \pm 0.1{ }^{\circ} \mathrm{C}$. Ambient relative humidities used for this study were $11,23,33,43,58$, $81,90,95$ and $97 \%$. The global water content $W$ were calculated from the mass of the samples. Note that the air velocity in the chamber in the vicinity of the sample was of about $2 \mathrm{~m} \cdot \mathrm{s}^{-1}$.

\subsubsection{Results}

Figures 1, 2 and 3 show the results of the measurements performed in adsorption (Fig. 1a, 2a and $3 a$ ) and in desorption (Fig. 1b, 2b and 3b) conditions for EASY-R, SHC and MHC respectively.

From Figure $1 \mathrm{a}, 2 \mathrm{a}$ and $3 \mathrm{a}$, in adsorption conditions, for $\mathrm{RH}$ lower or equal to $81 \%$, the stabilizations occur between 5 and 10 days. For higher $R H$, the stabilizations occur after several weeks, in some cases the measurements had been stopped before the stabilization in order to reduce the time of exposure and thus the risk of mold development. From Figure $1 b, 2 b$ and $3 b$, in desorption conditions the stabilizations are faster, less than 5 days for $R H$ lower than $81 \%$.

Figure $1 a, 1 b, 2 a, 2 b, 3 a$ and $3 b$

In (Collet et al. 2013), the measurements have been fitted by the following law:

$$
W(t)=W_{i}+a[1-b \exp (-c t)]
$$

where $W_{i}$ is the initial global water content of the sample and $a, b$ and $c$ are the fitting parameters. Thus, the equilibrium global water contents $W_{e q}$ are given by $W_{i}+a$. 


\subsubsection{Sorption isotherms}

The equilibrium water contents obtained from experiments have been reported in Figures 4, 5 and 6 for EASY-R, SHC and MHC respectively. Then, the sorption isotherms can be determined. In (Collet et al. 2013), they had been determined by fitting the measurements by the GAB model (Anderson and Hall 1948). In the present study, the Van Genuchten (1980) model has been used: contrary to the GAB model, the VG model is claimed valid even at high $R H(>90 \%)$. It is expressed as follow:

$$
w_{e q}(\varphi)=w_{\text {sat }}\left[1-(h \ln (\varphi))^{\eta}\right]^{1-1 / \eta}
$$

where $w_{\text {sat }}$ is the maximum equilibrium local water content (i.e. at $100 \% R H$ ) and $h$ and $\eta$ are adjustment coefficients.

Since global (i.e. at the sample scale) and local variables have the same values at the equilibrium, it can also be written:

$W_{e q}(R H)=W_{\text {sat }}\left[1-(h \ln (R H))^{\eta}\right]^{1-1 / \eta}$

Since the measurements in desorption conditions has been performed from a $R H$ of $97 \%$, this does not lead to the main isotherms of desorption. However, they can be determined by a combination of the Van Genuchten model and the hysteretic model of Huang et al. (2005) as done in (Oumeziane 2013; Oumeziane et al. 2014).

The isotherms of adsorption and desorption obtained for the three materials are shown in Figures 4, 5 and 6 and the values of the coefficients are given in Table 1: the adjustments has been performed in such a way that the agreements between the measurements and the model are the best possible for $R H$ greater than $30 \%$. Note that $w_{\text {sat }}$ has been determined assuming that the liquid water completely fills the open porosity.

Figures 4, 5 and 6

\begin{tabular}{|l|l|l|l|l|l|}
\hline & $W_{\text {sat }}\left(\mathrm{kg} \cdot \mathrm{m}^{-3}\right)$ & $h_{\text {ads }}(-)$ & $\eta_{\text {ads }}(-)$ & $h_{\text {des }}(-)$ & $\eta_{\text {des }}(-)$ \\
\hline EASY-R & 675 & 166 & 2.05 & 258 & 1.65 \\
\hline SHC & 660 & 378 & 1.77 & 500 & 1.46 \\
\hline MHC & 770 & 185 & 1.98 & 75 & 1.66 \\
\hline
\end{tabular}

Table 1: Van Genuchten model parameters obtained by adjustments for the three hemp concretes

\section{A model of local kinetics of sorption}

\subsection{Mathematical modeling}

\subsubsection{Governing equations}

In this section, (i) air transport is ignored and (ii) isothermal operating conditions are assumed. In the porous samples, water is present in gaseous form (water vapor) and in liquid form. Therefore, 
there are two mass balance equations to consider. Assuming that (iii) the convective transport is negligible, they take the following form:

$$
\left\{\begin{array}{l}
\frac{\partial \bar{\rho}_{v}}{\partial t}-\nabla \cdot\left(D_{p, v} \nabla \bar{\rho}_{v}\right)=-R_{s} \\
\frac{\partial \bar{\rho}_{l}}{\partial t}-\nabla \cdot\left(D_{p, l} \nabla \bar{\rho}_{l}\right)=R_{s}
\end{array}\right.
$$

where $\bar{\rho}_{v}$ and $\bar{\rho}_{l}$ are the local water vapor and liquid water partial densities, $D_{p, v}$ and $D_{p, l}$ are the water vapor and liquid water diffusivities and $R_{s}$ is the rate of sorption.

The local relative humidity can be expressed as follow:

$$
\varphi=\frac{P_{v}}{P_{\text {sat }}}=\frac{\bar{\rho}_{v} R T}{M_{w} P_{\text {sat }}}
$$

and the local liquid water partial density $\bar{\rho}_{l}$ (i.e. the local water content) will be written by convention as $w$.

Then, the mass balance equations can be rewritten as follow:

$$
\left\{\begin{array}{l}
\frac{\partial \varphi}{\partial t}-\nabla \cdot\left(D_{p, v} \nabla \varphi\right)=-\frac{R T}{M_{w} P_{s a t}} R_{s} \\
\frac{\partial w}{\partial t}-\nabla \cdot\left(D_{p, l} \nabla w\right)=R_{s}
\end{array}\right.
$$

It is commonly assumed that the rate of sorption $R_{s}$ is very fast compared to the vapor diffusive flux. According to this assumption, the sorption process is limited by the diffusive fluxes and $w$ moves along the isotherm of sorption $w_{e q}=w_{e q}(\varphi)_{\mid \text {| }}$. Therefore, we can write:

$$
\frac{\partial w}{\partial t}=\left.\frac{\partial w}{\partial \varphi}\right|_{T} \frac{\partial \varphi}{\partial t}, \text { and: } \nabla w=\left.\frac{\partial w}{\partial \varphi}\right|_{T} \nabla \varphi
$$

where $\partial w /\left.\partial \varphi\right|_{T}$ is given by the isotherm of sorption. From eqs. $(7,8,9,10)$, the following single governing equation can be obtained:

$\left.\frac{\partial w}{\partial \varphi}\right|_{T} \frac{\partial \varphi}{\partial t}-\nabla \cdot\left(D_{p, v} \frac{M_{w} P_{s a t}}{R T} \nabla \varphi+\left.D_{p, l} \frac{\partial w}{\partial \varphi}\right|_{T} \nabla \varphi\right)=0$

Introducing the material water vapor permeability $\delta_{v, p}$, it gives:

$$
\left.\frac{\partial w}{\partial \varphi}\right|_{T} \frac{\partial \varphi}{\partial t}-\nabla \cdot\left[\left(\delta_{v, p} P_{s a t}+\left.D_{p, l} \frac{\partial w}{\partial \varphi}\right|_{T}\right) \nabla \varphi\right]=0
$$

with: $\delta_{v, p}=\delta_{v} / \mu=D_{v} M_{w} / \mu R T$, the water vapor diffusion resistance factor being given by: $\mu=\delta_{v} / \delta_{v, p}=D_{v} / D_{p, v}$. 
Equation (12) is the so-called Künzel mass transfer equation (Künzel 1995) in its simplified form for isothermal conditions.

Now, assuming that the rate of sorption $R_{s}$ is not fast compared to the vapor diffusive flux, the two mass balance equations (3) and (4) must be considered. They can be re-written as follow:

$$
\left\{\begin{array}{l}
\frac{\partial \varphi}{\partial t}-\frac{R T}{M_{w}} \nabla \cdot\left(\delta_{v, p} \nabla \varphi\right)=-\frac{R T}{M_{w} P_{s a t}} R_{s} \\
\frac{\partial w}{\partial t}-\nabla \cdot\left(D_{p, l} \nabla w\right)=R_{s}
\end{array}\right.
$$

Then, the coupling with the hysteretic model of Huang et al. (2005) can easily be done: the reversal points (i.e. transitions between adsorption and desorption phases) are obtained when the sign of $R_{s}$ changes.

\subsubsection{Boundary conditions}

Considering a 1D cylindrical sample of radius $R_{a}$ placed in a chamber of relative humidity $R H$, the relative humidity at its circumferential edge is given by:

$$
\varphi\left(r=R_{a}\right)=R H-\left.\frac{\delta_{v, p}}{h_{m}} \cdot \frac{\partial \varphi}{\partial r}\right|_{r=R_{a}}
$$

where $h_{m}$ is the mass transfer coefficient $\left(\mathrm{kg} \cdot \mathrm{m}^{-2} \cdot \mathrm{Pa}^{-1} \cdot \mathrm{s}^{-1}\right)$.

With the classic approach of Künzel, there is a single governing equation and there is no need to define a water content boundary condition. However, it is implicitly given by:

$$
\left.\frac{\partial w}{\partial r}\right|_{r=R_{a}}=\left.\left.\frac{\partial w}{\partial \varphi}\right|_{T} \frac{\partial \varphi}{\partial r}\right|_{r=R_{a}}
$$

Under the assumption of a slow sorption rate $R_{s}$, we can write:

$$
\left.\frac{\partial w}{\partial r}\right|_{r=R_{a}}=\left.\left.\beta \frac{\partial w}{\partial \varphi}\right|_{T} \frac{\partial \varphi}{\partial r}\right|_{r=R_{a}}
$$

where $B$ is in the range of 0 to 1 . Its value will be considered as a constant ( 0 or 1$)$, actually it dynamically depends on $R_{s}$.

\subsection{Preliminary simulations and proposal of a sorption rate}

Figure 7 shows the global kinetics of adsorption of the sample of EASY-R initially stabilized at a $R H$ of $33 \%$ and submitted to an ambient $R H$ of $43 \%$. From the measurements (Collet et al. 2013), the stabilization of the global water content $W$ of the sample only occurs after 7 to 10 days.

According to the classic assumption of instantaneous local equilibrium between relative humidity and water content (according to the adsorption isotherms), the 1D cylindrical calculation (i.e. with the Künzel equation) leads to a stabilization lower than 1 day (Fig. 7). Still with this assumption, a water vapor resistance vapor $\mu$ of 65 should be considered (instead of its measured value of 4 ) to properly 
reproduce the temporal evolution (Fig. 7). That does not make any sense and clearly shows that the classic assumption of instantaneous local equilibrium between relative humidity and water content is not valid in these operating conditions.

Figure 7

Therefore, a relatively low kinetics of sorption may locally occur. It has already been noticed for different bio-based porous material such as wood (Frandsen et al. 2007; Eitelberger and Svensson 2011) or paper (Alexandersson et al. 2016) and various cellulosic materials (Nyman et al. 2006). It may be presumed that this finding is generalizable whatever the bio-based porous material. But not only this class of materials is concerned since some studies show that it is also the case for more "classic" compounds such as cement based porous materials (Johannesson and Nyman 2010; Zeng et al. 2015). It has also been evidenced for soils (Maraqa 2016).

A kinetics is usually expressed as a kinetic constant multiplied by a driving force. Some studies have proposed to express the driving force as a function of local relative humidities with more or less success (Frandsen et al. 2007; Eitelberger and Svensson 2011; Alexandersson et al. 2016; Maraqa 2016). The problem is that they consider an equilibrium relative humidity which is a priori not known for a local approach and/or they have to change the value of the kinetics constant according the operating conditions. Actually, it seems more natural to express the driving force as a function of the local water content and thus the simplest expression for the sorption rate is the following:

$R_{s}=k_{0}\left(w_{e q}(\varphi)-w\right)$

where $k_{0}$ is the local kinetic constant of sorption (adsorption or desorption) and $w_{e q}$ is the equilibrium local water content given by the sorption isotherm at a local relative humidity $\varphi$.

Nyman et al. (2006) and Johannesson and Nyman (2010) came to use the same expression. However, the value of the kinetic constant $k_{0}$ still have to be adjusted as a function of the operating conditions: this is not really satisfactory, the local kinetic constant should only depend on the porous materials and not on the hygric conditions, at least as long as the hygric state is far from saturation.

Consequently, a more complex driving force is considered in our study introducing a kinetic order $n$ :

$R_{s}=k_{0}\left(w_{e q}(\varphi)-w\right)^{n}$

Thus, a new calculation based on the local kinetics assumption has been performed adjusting the parameters $k_{0}$ and $n$. As shown in Figure 7, experiment is properly reproduced using a value of $k_{0}$ of 2 $\mathrm{day}^{-1} /\left(\mathrm{kg} \cdot \mathrm{m}^{-3}\right)$ and a kinetic order $n$ of 2 . Note that the best adjustment is indeed obtained with these values.

Note that with the value of $h_{m}$ considered here, i.e. $7 \cdot 5 \cdot 10^{-8} \mathrm{~kg} \cdot \mathrm{m}^{-2} \cdot \mathrm{Pa}^{-1} \cdot \mathrm{s}^{-1}$, the results are extremely close to the ones which would be obtained considering: $\varphi(r=R)=R H$. Moreover, calculations performed with values of $B$ of 0 and of 1 lead to extremely close results and the same adjustments of the kinetic parameters.

\section{Simulations}

4.1 Preliminary considerations about the mass transfer coefficient $h_{m}$ 
An important remark has to be made about the value of the mass transfer coefficient $h_{m}$ which has an effect of the relative humidity boundary conditions at the surface of the sample (eq. 11). The value of $h_{m}$ is typically in the range of $2.10^{-8}$ to $2 \cdot 10^{-9} \mathrm{~kg} \cdot \mathrm{m}^{-2} \cdot \mathrm{Pa}^{-1} \cdot \mathrm{s}^{-1}$ depending on the operating conditions and on the studied material. It is worth to note that modeling studies of the literature about hygrothermal transfers tend to avoid to discuss this point considering a given value of $h_{m}$ without legitimate justification.

From in-depth studies (Mortensen et al. 2005; Talev et al. 2012), for a chamber in which mean air velocity is about $2 \mathrm{~m} \cdot \mathrm{s}^{-1}$, the value of $h_{m}$ is of about $5.10^{-8} \mathrm{~kg} \cdot \mathrm{m}^{-2} \cdot \mathrm{Pa}^{-1} \cdot \mathrm{s}^{-1}$. Moreover, a multiplication factor has to be considered taking into account the rugosity of the sample. It is usually estimated of about 1.5 or even 2 for concretes (Oumeziane 2013). It leads to a value of $h_{m}$ of about $7.5 .10^{-8} \mathrm{~kg} \cdot \mathrm{m}^{-}$ ${ }^{2} \cdot \mathrm{Pa}^{-1} \cdot \mathrm{s}^{-1}$.

As aforementioned, with such a value of $h_{m}$, the results are extremely close to the ones which would be obtained considering: $\varphi(r=R)=R H$. Actually, a significant effect would begin to be noticed in the very first hours of the sorption process for a ten times lower value of $h_{m}$. Therefore, for the present study, focused on day/week time scales, $h_{m}$ is not a sensitive parameter.

\subsection{Hemp concrete EASY-R}

The calculations based on the local kinetics assumption have been performed adjusting the parameters $k_{0}$ and $n$. A constant value of $\mu$ of 4 has been considered and the liquid diffusivity has been ignored. As evidenced by the results reported in Figure 1a, still considering a value of $k_{0}$ of 2 day $1 /\left(\mathrm{kg} \cdot \mathrm{m}^{-3}\right)$ and a kinetic order $n$ of 2 , the adjustments are globally very good.

If we consider instead a kinetic order of 1 , the adjustments are less good and the values obtained for $k_{0}$ differ by a factor 3 depending on the hygric conditions.

A sensitivity study on $k_{0}$ has been performed for the $33 \%$ to $43 \% R H$ step. $W$ has been considered as stabilized as soon as it is equal to $97 \%$ of its final value. It leads to durations of stabilization equal to $14.5,7.8,5.6$ and 4.4 days for $k_{0}$ equal to $1,2,3$ and $4 \mathrm{day}^{-1} /\left(\mathrm{kg}^{-3} \mathrm{~m}^{-3}\right)$ respectively. Therefore, the duration of stabilization is broadly inversely proportional to the value of $k_{0}$.

Moreover, for $R H$ lower than $80 \%$, whatever the value of $\mu$ between 3 and 5 , the best adjustments are always obtained with a value of $k_{0}$ of $2 \mathrm{day}^{-1} /\left(\mathrm{kg} \cdot \mathrm{m}^{-3}\right)$ : in this range, $\mu$ has an insignificant effect on the adjustment of $k_{0}$.

Still under these hygric conditions $(R H<80 \%)$, the vapor diffusion process is very fast (and bring vapor in sufficient high quantities) compared to the local kinetics. Therefore, $k_{0}$ is the only limiting factor of the sorption process, the duration of the stabilization should not depend of the sample size theoretically (provided that the sample size is representative).

For $\mathrm{RH}$ higher than $80 \%$, the times of stabilization are longer: the water vapor mass transfer becomes a limiting factor as free liquid water appears because it takes a while for the diffusion process to bring such high quantities of water required to reach the moisture equilibrium. Actually, for these hygric conditions, both $\mu$ and $k_{0}$ are limiting factors. Thus, under these hygric conditions, the more important the sample size is, the longer the duration of stabilization is.

Note that for these operating conditions, taking $b=1$ or 0 for the water content boundary condition of the exposed surface does not lead to perceptible differences. 
Then, Figure $1 \mathrm{~b}$ shows the measurements of the global kinetics of desorption for gradual $R H$ steps between $90 \%$ and $23 \%$. Still considering a value of $k_{0}$ of $2 \mathrm{day}^{-1} /\left(\mathrm{kg} \cdot \mathrm{m}^{-3}\right)$ and a kinetics order $n$ of 2 , the adjustments of the calculations are still globally very good.

Overall, the correlation coefficients are between 0.975 and 0.995 as long as the relative humidity remains greater than $23 \%$. They could be even better if the experimental points were less dispersed (this is quite noticeable in Fig. 7). In the range of 0 to $23 \% R H$, the climatic chamber regulation is poor and therefore the experimental data are not reliable.

\subsection{Hemp concrete SHC}

The calculations based on the local kinetics assumption have been performed adjusting the parameters $k_{0}$ and $n$. A constant value of $\mu$ of 4 has been considered and the liquid diffusivity has been ignored. As evidenced by the results reported in Figure 2a (adsorption) and Figure $2 b$ (desorption), considering a value of $k_{0}$ of $0.65 \mathrm{day}^{-1} /\left(\mathrm{kg} \cdot \mathrm{m}^{-3}\right)$ and a kinetic order $n$ of 2 , the adjustments are globally very good.

\subsection{Hemp concrete MHC}

The calculations based on the local kinetics assumption have been performed adjusting the parameters $k_{0}$ and $n$. A constant value of $\mu$ of 4 has been considered and the liquid diffusivity has been ignored. As evidenced by the results reported in Figure 3a (adsorption) and Figure 3b (desorption), considering a value of $k_{0}$ of $0.5 \mathrm{day}^{-1} /\left(\mathrm{kg} \cdot \mathrm{m}^{-3}\right)$ and a kinetic order $n$ of 2 , the adjustments are globally very good.

\subsection{Discussion}

For these three hemp concretes, the best adjustments are obtained with a second order kinetics. Although these three materials are similar in terms of composition, the kinetic constant obtained by adjustments are not the same. It appears that the higher $k_{0}$ is obtained for EASY-R which has the higher hemp/binder mass ratio: its higher proportion of hemp shiv might allow an easier accessibility for the water molecules to the trapped porosity inside the mineral matrix. The distributions of the porous networks might plays a key role though. It is likely that the three different manufacturing processes lead to significantly different porous networks.

For bio-based materials, the origin of the local kinetics might be explained by the existence of bound water (Frandsen et al. 2007; Engelund and Thygesen 2012). Once in liquid state, the liquid water molecules enter into the biological cells. Bound water molecules slowly diffuse in these cells and finally fill the porosities trapped between/around these cells and/or inside the matrix. Therefore, the apparent local kinetics might be ruled by the slowness of bound water diffusion. From (Christensen 1959; Engelund and Thygesen 2012), this kind of diffusion induces mechanical strains in the samples and its speed would actually be linked to the speed of relaxation of these mechanical strains. As aforementioned, the distributions of the porous networks may play a role too. Therefore, it seems that the origin of the local kinetics is extremely complex with an interdependence of hygric transport and mechanics, this question would require further long and deep investigations and then the kinetic order of 2 might be physically justified. 
Note that in ordinary concretes, there is no biological cells but there are probably significant chemical interactions between the water molecules and the pores walls in the nano/micro-porous networks (Zeng and Xu 2017). These chemical interactions can also result, to some extent, in "bound" water. Nevertheless, since ordinary concretes are usually much less porous than the hemp concretes studied here, the diffusion transport might be a limiting factor compared to the local kinetics.

As aforementioned, for the hemp concretes studied here, the sorption mechanism is clearly limited by the kinetics at low $R H(<80 \%)$. At higher $R H$, the mass transport is also involved. For these high $R H$ and water contents, the kinetics may actually decrease due to the decrease of the accessible surface to the sorption, the interface area solid/water vapor being smaller and smaller due to free water-logging. This may be compensated by the free liquid water transport by capillarity (driven by the liquid diffusivity) which may become significant. From the modeling studies of (Oumeziane 2013; Oumeziane et al. 2014) performed over the basis of permeability measurements of (Collet et al. 2013), the liquid diffusivity in EASY-R samples initiates from $R H$ of about $60 \%$ and can be expressed as follow:

$$
\left\{\begin{array}{l}
D_{p, l}=0 \text { for } \varphi \leq 0.6 \\
D_{p, l}=4.10^{-10} \exp \left(5.8 .10^{-2} w\right)-5.83 .10^{-10} \text { for } \varphi>0.6
\end{array}\right.
$$

considering that $\mu$ remains constant and that the increase of permeability as a function of water content is due to the increase of liquid diffusivity. The simulations for EASY-R have been redone taking into account this liquid diffusivity law for $R H$ greater than $60 \%$ and the values of $k_{0}$ have been readjusted. For the steps $58 \%$ to $81 \% R H, 81 \%$ to $90 \% R H, 90 \%$ to $95 \% R H$ and $95 \%$ to $97 \% R H$, it leads to values of $k_{0}$ of $1.5,0.1,0.02$ and 0.002 respectively. For the three last cases, the results of the adjustments have been reported in Figure 8 and can be compared to measurements and previous calculations. It appears that the adjustments are very significantly better from $R H$ greater than $90 \%$. It means that at high $R H$ and $w$, near saturation, there would be an exponential decrease of the kinetic constant compensated by an exponential increase of the liquid diffusivity.

Finally, further investigations will have to be performed to verify whether the proposed local kinetics model is still valid in the very first hours of the sorption process.

\section{A semi-empirical model for kinetic parameters identification}

Evaluation of the local kinetic parameters requires sufficient experimental data. Empirical models used to describe the kinetics of sorption may be helpful to limit the needed data. From (Brown et al. 1980) and from the study of (Nguyen 2009) focusing on the hydration / dehydration of hydrated minerals, the global kinetics of water adsorption / desorption of a sample can be well described by the following empirical proposed expression given in Table 3.2 of (Nguyen 2009):

$$
\frac{d \alpha}{d t}=K(1-\alpha)^{p} \alpha^{q}
$$

with: $\alpha=\frac{W(t)-W_{i}}{W_{f}-W_{i}}$,

where $W_{i}$ and $W_{f}$ are the initial and final global water contents of the sample, $K$ is a global kinetic factor and the exponent $p$ and $q$ are adjustable parameters. $\alpha$ represents the proportion of bound and/or trapped water. Assuming that $q$ is a zero coefficient, this global model can be re-written as follow: 


$$
W(t)=W_{i}+\left(W_{f}-W_{i}\right) \frac{\left(1+K_{0} t\right)^{\frac{1}{p-1}}-1}{\left(1+K_{0} t\right)^{\frac{1}{p-1}}}
$$

where $K_{0}$ is a first order global kinetic constant of adsorption or desorption. Under strong assumptions, this expression with a parameter $p$ of 2 is actually an approximate solution of equations $(14,19)$ with a kinetic order $n$ of 1 . Therefore, this expression is at least a semi-empirical model:

$W(t)=W_{i}+\left(W_{f}-W_{i}\right) \frac{K_{0} t}{1+K_{0} t}$

Or, in an even simplest form:

$W(t)=\frac{W_{i}+W_{f} K_{0} t}{1+K_{0} t}$

For the materials studied here, it appears that this law allows much better adjustments than the traditionally used law (eq. 1).

Note that in the study (Zengh and Xu 2017), the authors use and validate, for cement compounds, a more complex form of this kind of model since $t$ is raised to $t^{\delta}, \delta$ being the so-called time index. The so-called FK (fractional kinetic) model is based on the work of Brouers and Sotolongo-Costra (Brouers and Sotolongo-Costa, 2006).

However, in the expression of this kind of models, $K_{0}\left(\right.$ day $^{-1}$ or day $\left.^{-\delta}\right)$ is a first order kinetic constant. As shown in the previous section, the kinetic order $n$ to consider should actually be of 2 . Thus, the following modified expression, assuming a kinetic order $n$ of 2, can now be proposed:

$W(t)=\frac{W_{i}+W_{f}\left(W_{f}-W_{i}\right) K_{0} t}{1+\left(W_{f}-W_{i}\right) K_{0} t}$

where $K_{0}$ is the adjustable global kinetic constant expressed in day $^{-1} /\left(\mathrm{kg} \cdot \mathrm{m}^{-3}\right)$.

Now, adjusting this model on the $33 \%$ to $43 \% R H$ steps, we obtain the following values of $K_{0}: 1.75,0.6$ and $0.45 \mathrm{day}^{-1} /\left(\mathrm{kg}_{\mathrm{g}} \mathrm{m}^{-3}\right)$ for EASY-R, SHC and MHC respectively. This is quite close to the values of the local kinetic constant $k_{0}$ deduced from the 1D cylindrical calculations.

In Figure 9, we can compare the temporal evolutions of $W$ obtained with the 1D cylindrical calculations and this semi-empirical model for EASY-R, 33\% to $43 \%$ RH step: the curves are very close.

Figure 9

Now, for EASY-R, still for the $33 \%$ to $43 \% R H$ step, if the semi-empirical model is straightly adjusted on the curve obtained with the 1D cylindrical calculations, a value of $K_{0}$ of 1.86 is obtained (instead of 2 from the $1 D$ calculation).

These results are very interesting because it means that the kinetic constants can be straightly deduced from the adjustment of an analytical expression (eq. 26) instead from 1D cylindrical calculations. It works provided the studied range of $\mathrm{RH}$ remains between $23 \%$ and $80 \%$ (above $80 \%$, what is perceived as kinetics could actually be liquid diffusion, as explained in the previous section). 


\section{Conclusion}

For the three hemp concrete studied, 1D cylindrical simulations have shown that the classic assumption of instantaneous $\varphi / \mathrm{w}$ equilibrium according to the sorption isotherms leads to serious inconsistencies compared to experiments. An expression of a local kinetics of sorption with a kinetic order of 2 has been proposed and validated against experiments for the three studied hemp concretes.

Unlike the studies available in literature, it has been possible to find values of the kinetic constant really constant for each of the three materials, whatever the hygric conditions. However, deeper investigations have suggested that, near saturation, there is actually an exponential decrease of the kinetic constant compensated by an exponential increase of the liquid water diffusivity.

Finally, a semi-empirical analytical model has been proposed to estimate the value of the kinetic constant from the sorption test data. This way is easier and faster than performing simulations.

This work seriously calls into question the classic assumption of instantaneous $\varphi / w$ equilibrium considered in the commercial simulation tools used in the building industry. But besides this sector, this work may also be useful in all the domains for which hygric transfers in porous media is of main concern, e.g. food industry or soils.

In further investigations, the effect of the local kinetics model on the hygrothermal transfers occurring through bio-based walls will be studied. The case of cyclic hygrothermal exposures will be full of interests.

\section{References}

Alexandersson, M., Askfelt, H., Ristinmaa, M.: Triphasic model of heat and moisture transport with internal mass exchange in paperboard. Transp. Porous Med. 112, 381-408 (2016)

Anderson, R.B., Hall, W.K.: Modifications of the Brunauer, Emmett and Teller, equation II. J. Amer. Chem. Soc. 70 (5), 1727-1734 (1948)

Bouers, F., Sotolongo-Costa, O.: Generalized fractal kinetics in complex systems (application to biophysics and biotechnology). Phys. A 368, 165-175 (2006)

Brown, M.E., Dollimore, D., Galwey, A.K.: Reactions in the solid state. In: Bamford, C.H., Tiper, C.F.H. (eds) Comprehensive Chemical Kinetics, Vol. 22, pp. 340, Elsevier, Amsterdam (1980)

Carmeliet, J., De Wit, M., Janssen, H.: Hysteresis and moisture buffering of wood. $7^{\text {th }}$ Nordic Symposium on Building Physics, Reykjavik, Islande (2005)

Chamoin, J.: Optimisation des propriétés (physiques, mécaniques et hydriques) de bétons de chanvre par la maîtrise de la formulation. PhD thesis, Génie civil, INSA de Rennes (2013)

Collet, F., Chamoin, J., Pretot, S., Lanos C.: Comparison of the hygric behaviour of three hemp concretes. Energy and Buildings 62, 294-303 (2013)

Christensen, G.N.: The rate of sorption of water vapour by wood and pulp. Appita J. 13, 112-123 (1959)

Eitelberger, J., Svensson, S.: The sorption behavior of wood studied by means of an improved cup method. Transp. Porous Med. 92, 321-335 (2012) 
Engelund, E.T., Thygesen, L.G., Svensson S., Hill, C.A.S.: A critical discussion of the physics of woodwater interactions. Wood Sci. Technol. 47, 141-161 (2013)

Frandsen, H.L., Damkilde, L., Svensson, S.: A revised multi-Fickian moisture transport model to describe non-Fickian effects in wood. Holzforschung, Vol. 61, pp. 563-572, Copyright by Walter de Gruyter, Berlin, New York (2007)

Huang, H.C., Tan, Y.C., Liu, C.W., Chen C.H.: A novel hysteresis model in unsaturated soil. Hydrological Processes 19(8), 1653-1665 (2005)

Iglesias, H.A., Chirife, J.: Handbook of Food Isotherms: Water Sorption Parameters for Food and Food Components. Academic Press Inc., United Kingdom Edition (1982)

Johannesson, B., Nyman, U.: A numerical approach for non-linear moisture flow in porous materials with account to sorption hysteresis. Transp. Porous Med. 84, 735-754 (2010)

Kumaran, M.: Interlaboratory Comparison of the ASTM Standard Test Methods for Water Vapor Transmission of Materials (E 96-95). J. Test. Eval. 26(2), 83-88 (1998)

Künzel, H.M.: Simultaneous Heat and Moisture Transport in Building Components - One- and TwoDimensional Calculation Using Simple Parameters, Fraunhofer IRB Verlag Suttgart, ISBN 3-8167-41037 (1995)

Maqara, M.A.: Ability of single-rate models to predict solute distribution coefficients in systems with heterogeneous sorption kinetics. Transp. Porous Med. 112, 765-781 (2016)

Mortensen, L.H., Rode, C., Peuhkuri, R.: Effect of airflow velocity on moisture exchange at surfaces. BYG-DTU, Trondheim, October 26-28 (2005)

Nguyen K.-S. : Comportement thermos-chimique de matériaux minéraux : application à la protection incendie. PhD thesis, LGCGM, Univ Rennes (2009)

Nyman, U., Gustafsson, P.J., Johannesson, B., Hägglund, R.: Numerical method for the evaluation of non-linear transient moisture flow in cellulosic materials. Int. J. Numer. Meth. Engng 66, 1859-1883 (2006)

Oumeziane, Y.A.: Evaluation des performances hygrothermiques d'une paroi par simulation numérique : application aux parois en béton de chanvre. PhD thesis, Génie civil, INSA de Rennes (2013)

Oumeziane, Y.A, Bart, M., Moissette S., Lanos C.: Hysteretic behaviour and moisture buffering of hemp concrete. Transp. Porous Med. 103, 515-533 (2014)

Talev, G., Jelle, B.P., Næss, E., Gustavsen, A., Thue J.V.: Measurement of the convective moisture transfer coefficient from porous building material surfaces applying a wind tunnel method. Journal of Building Physics 37(1), 103-121 (2012)

Van Genuchten, M.Th.:A closed-form equation for predicting the hydraulic conductivity of unsatured soils. Soil Science Society American Journal 4, 892-898 (1980)

Zeng, Q., Zhang, D., Li K.: Kinetics and equilibrium isotherms of water vapor adsorption/desorption in cement-based porous materials. Transp. Porous Med. 109, 469-493 (2015)

Zengh, Q., Xu, S.: A two-parameter stretched exponential function for dynamic water vapor sorption of cement-based porous materials. Mater. Struct. 50:128 (2017) 


\section{Figures captions}

Gnuplot software has been used to create the artwork

Fig. 1 Temporal evolution of the global water content $W$ in a sample of EASY-R for (a) increasing / (b) decreasing $R H$ steps - Experiments (points) and simulations (lines) with $\mu=4, k_{0}=2 \mathrm{day}^{-1} /\left(\mathrm{kg}^{-\mathrm{m}^{-3}}\right)$ and $n=2$.

Fig. 2 Temporal evolution of the global water content $W$ in a sample of SHC for (a) increasing / (b) decreasing $R H$ steps - Experiments (points) and simulations (lines) with $\mu=4, k_{0}=0.65 \mathrm{day}^{-1} /\left(\mathrm{kg} \cdot \mathrm{m}^{-3}\right)$ and $n=2$.

Fig. 3 Temporal evolution of the global water content $W$ in a sample of MHC for (a) increasing / (b) decreasing $R H$ steps - Experiments (points) and simulations (lines) with $\mu=4, k_{0}=0.5 \mathrm{day}^{-1} /\left(\mathrm{kg}^{-\mathrm{m}^{-3}}\right)$ and $n=2$.

Fig. 4 Isotherms of adsorption / desorption obtained from experiments (points) at $T=23^{\circ} \mathrm{C}$ for EASY$R$ and fitted by the VG - VG/Huang models (lines)

Fig. 5 Isotherms of adsorption / desorption obtained from experiments (points) at $T=23^{\circ} \mathrm{C}$ for SHC and fitted by the VG - VG/Huang models (lines)

Fig. 6 Isotherms of adsorption / desorption obtained from experiments (points) at $T=23^{\circ} \mathrm{C}$ for MHC and fitted by the VG - VG/Huang models (lines)

Fig. 7 Temporal evolution of the global water content $W$ of a sample of EASY-R stabilized at $33 \% R H$ and submitted to an ambient $R H$ of $43 \%$ - Experiments, simulations with the equilibrium model with $\mu=4 / \mu=65$, simulation with the local kinetics model with $\mu=4, k_{0}=2$ day $^{-1} /\left(\mathrm{kg} \cdot \mathrm{m}^{-3}\right)$ and $n=2$.

Fig. 8 Temporal evolution of the global water content $W$ in a sample of EASY-R for increasing $R H$ steps - Experiments (points), simulations with no liquid diffusivity (lines) and with liquid diffusivity (dashed lines)

Fig. 9 Temporal evolution of the global water content $W$ in a sample of EASY-stabilized at $33 \% R H$ and submitted to an ambient $R H$ of $43 \%$ - Experiments (points), simulation with $k_{0}=2 \mathrm{day}^{-1} /\left(\mathrm{kg} \cdot \mathrm{m}^{-3}\right)$ and $n=2$ (line) and semi-empirical model with $K_{0}=1.75 \mathrm{day}^{-1} /\left(\mathrm{kg} \cdot \mathrm{m}^{-3}\right)$ (dashed line) 

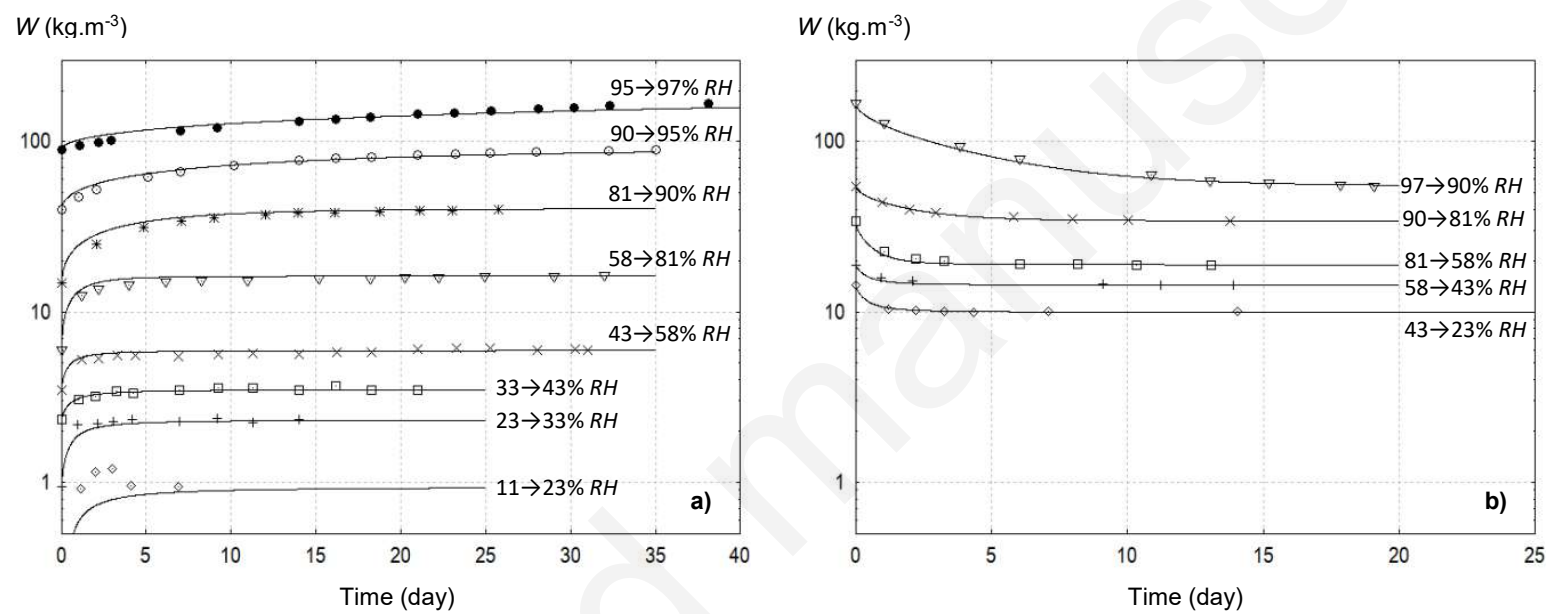

Fig. $1 a$ and $1 b$ 
$W\left(\mathrm{~kg} \cdot \mathrm{m}^{-3}\right)$

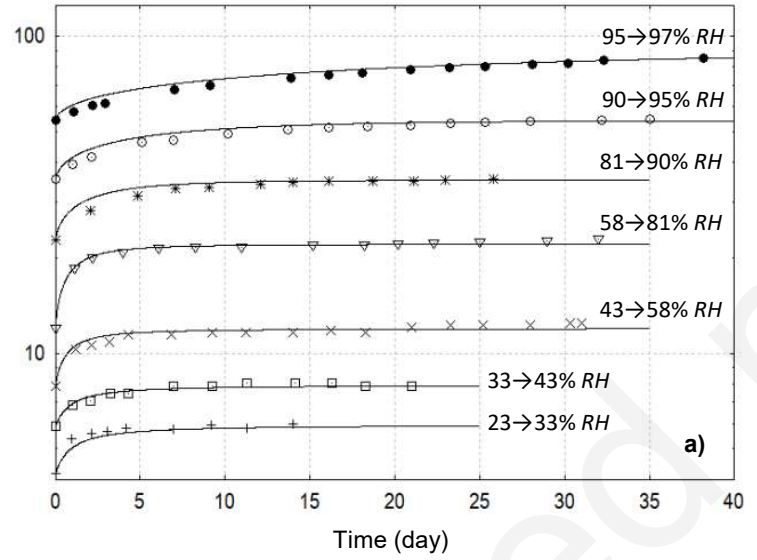

$W\left(\mathrm{~kg} \cdot \mathrm{m}^{-3}\right)$

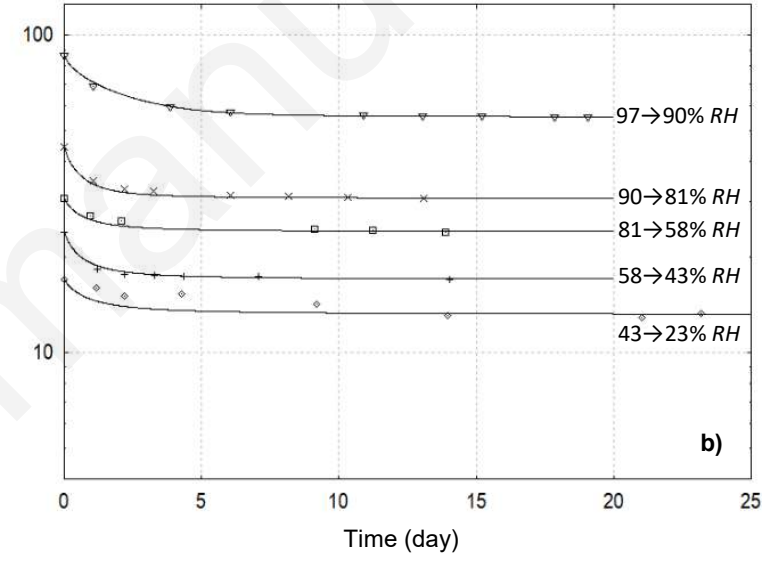

Fig. $2 a$ and $2 b$ 

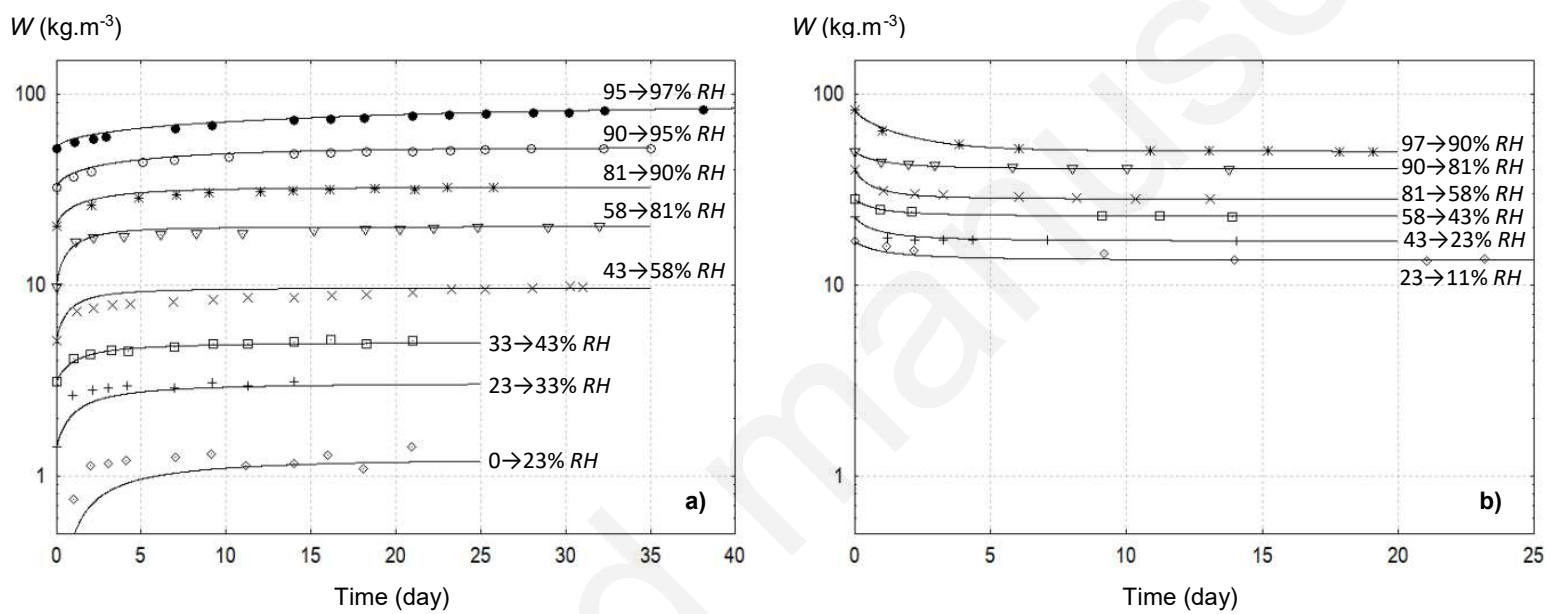

Fig. $3 a$ and $3 b$ 


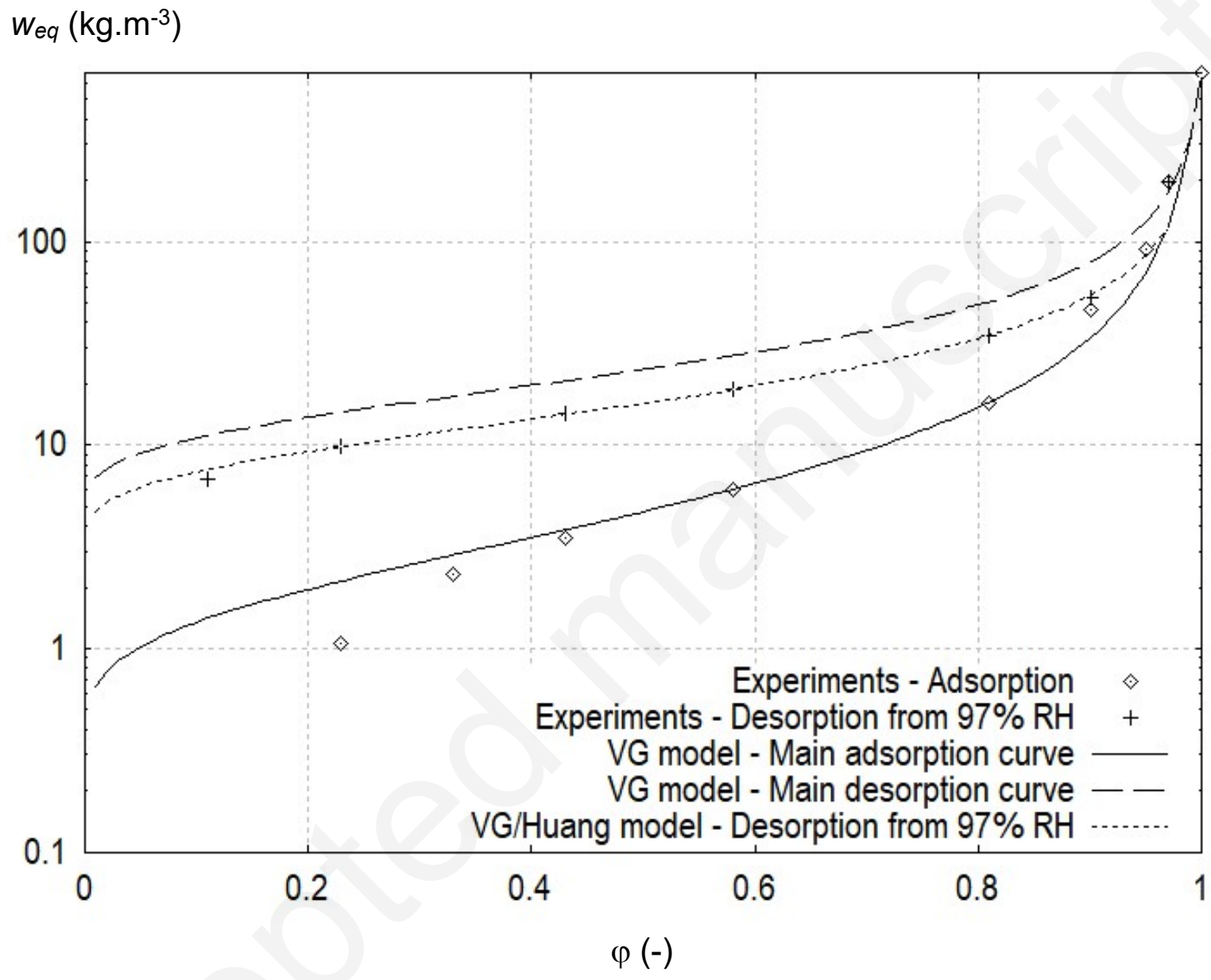

Fig. 4 


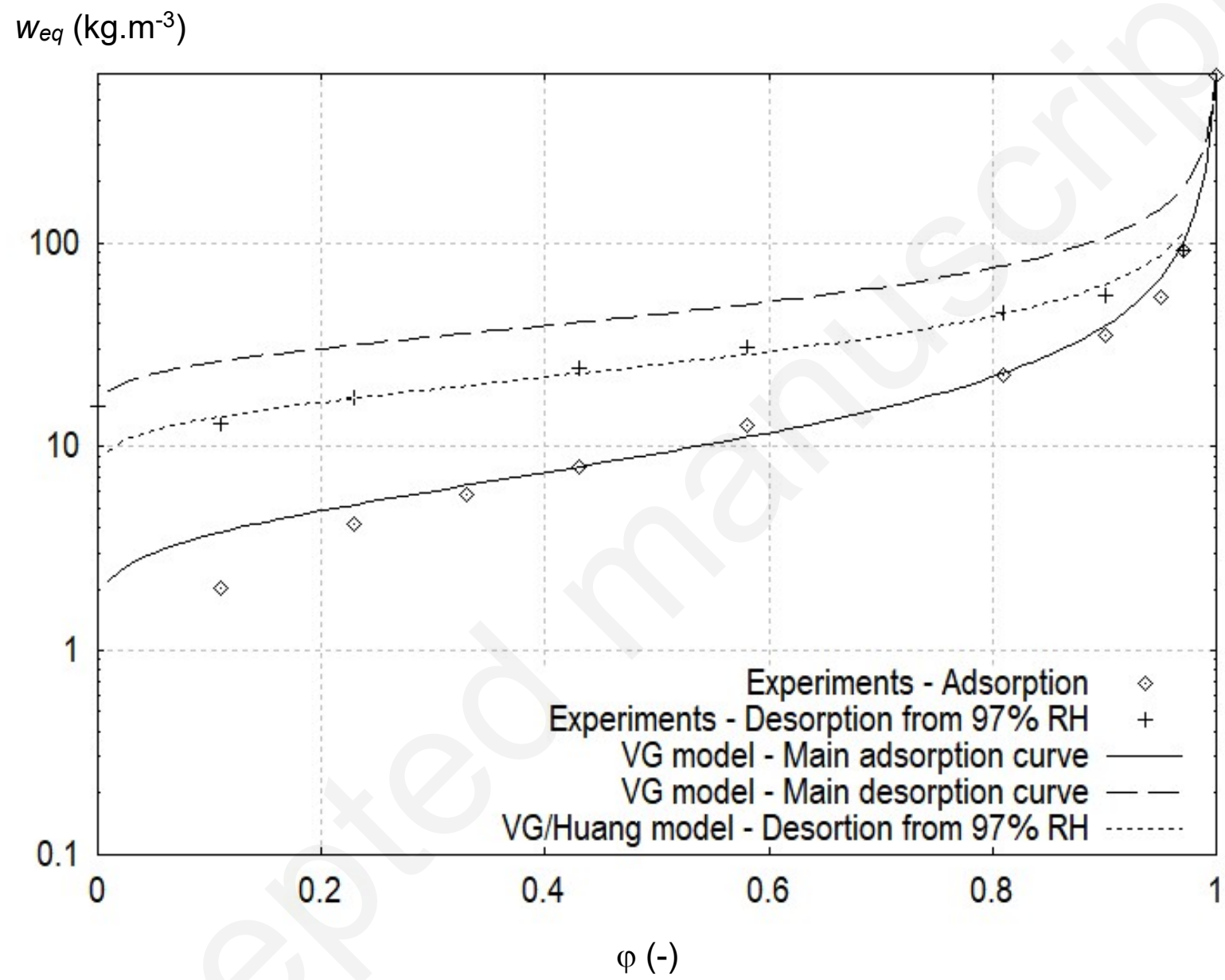

Fig. 5 


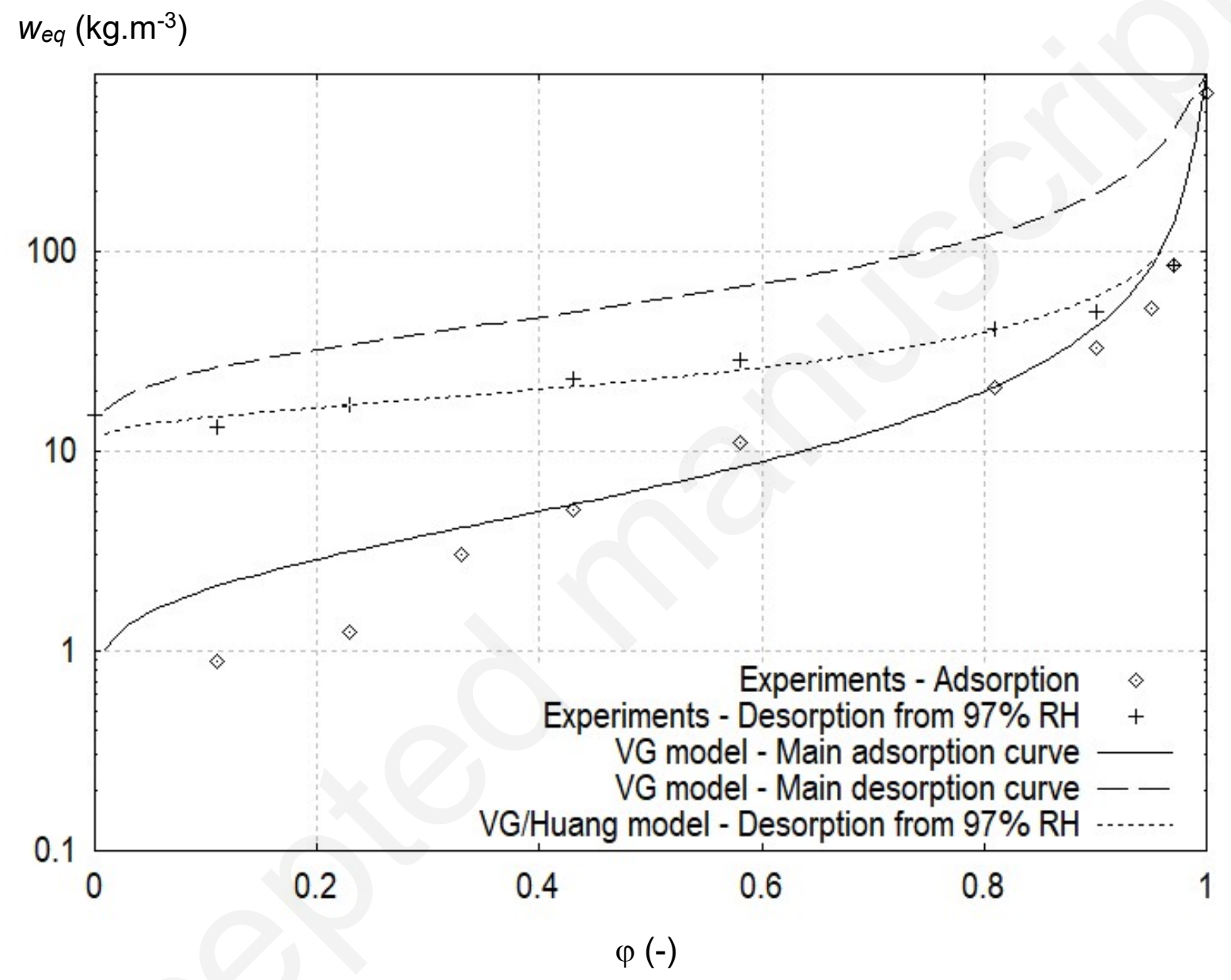

Fig. 6 


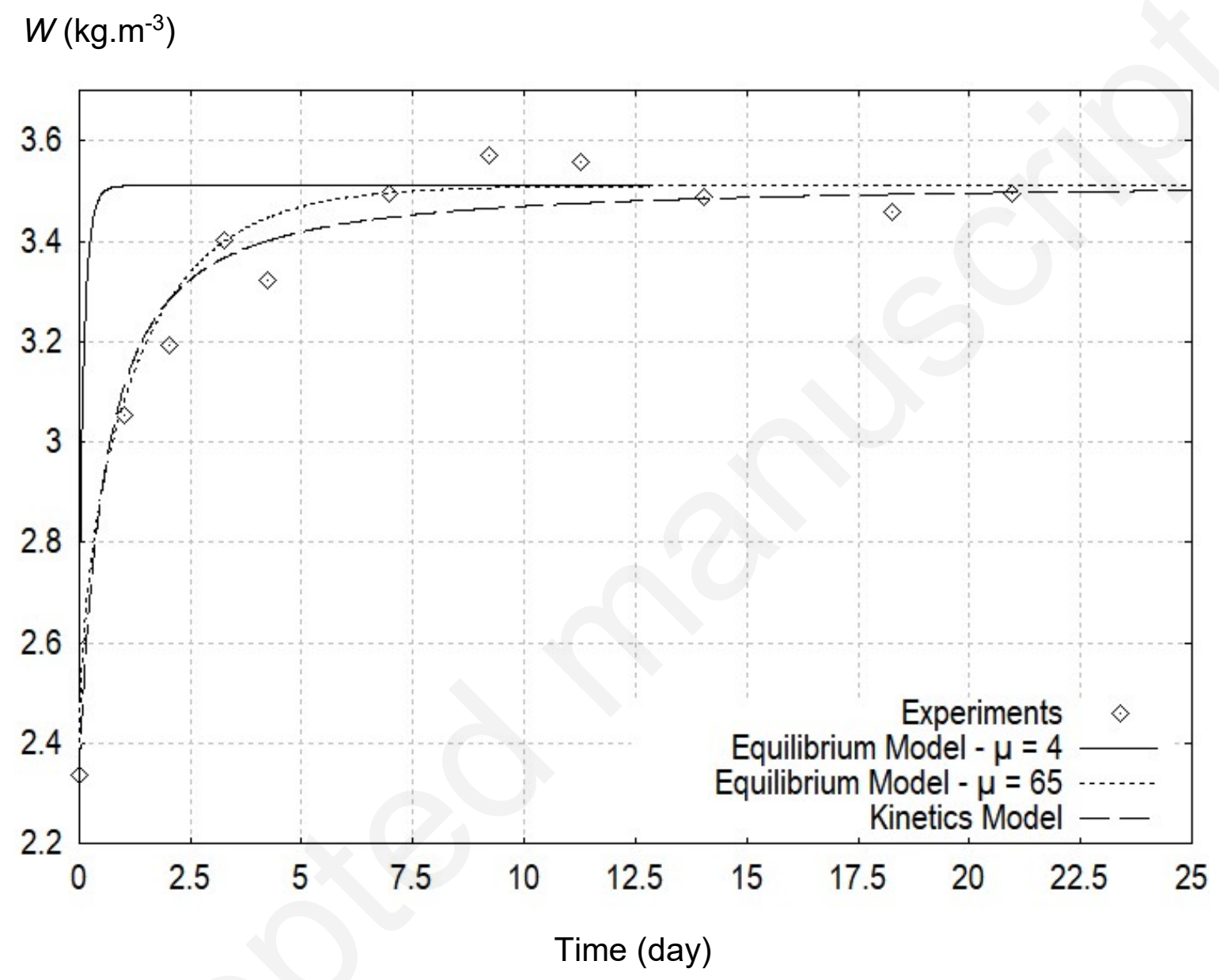

Fig. 7 


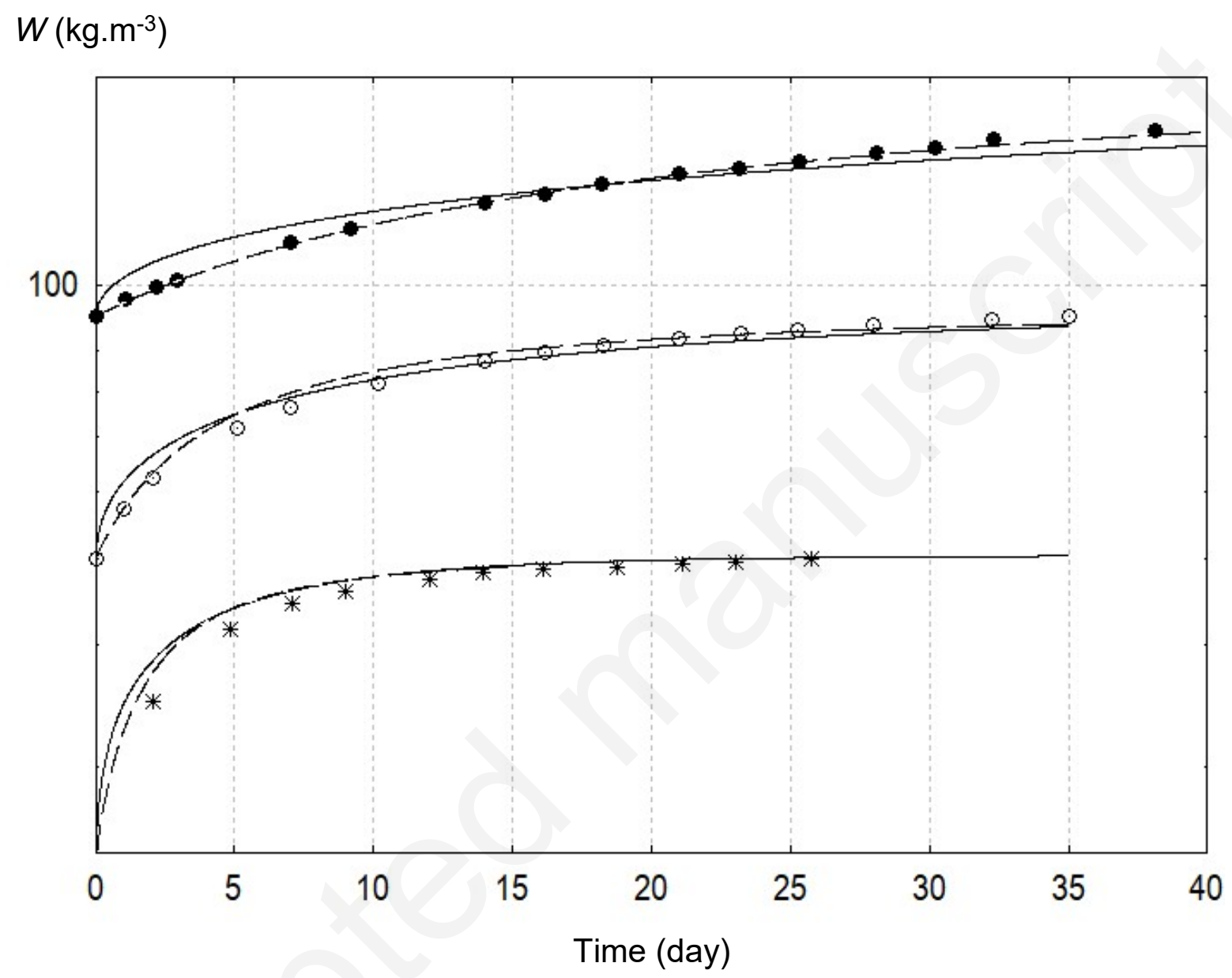

Fig. 8 


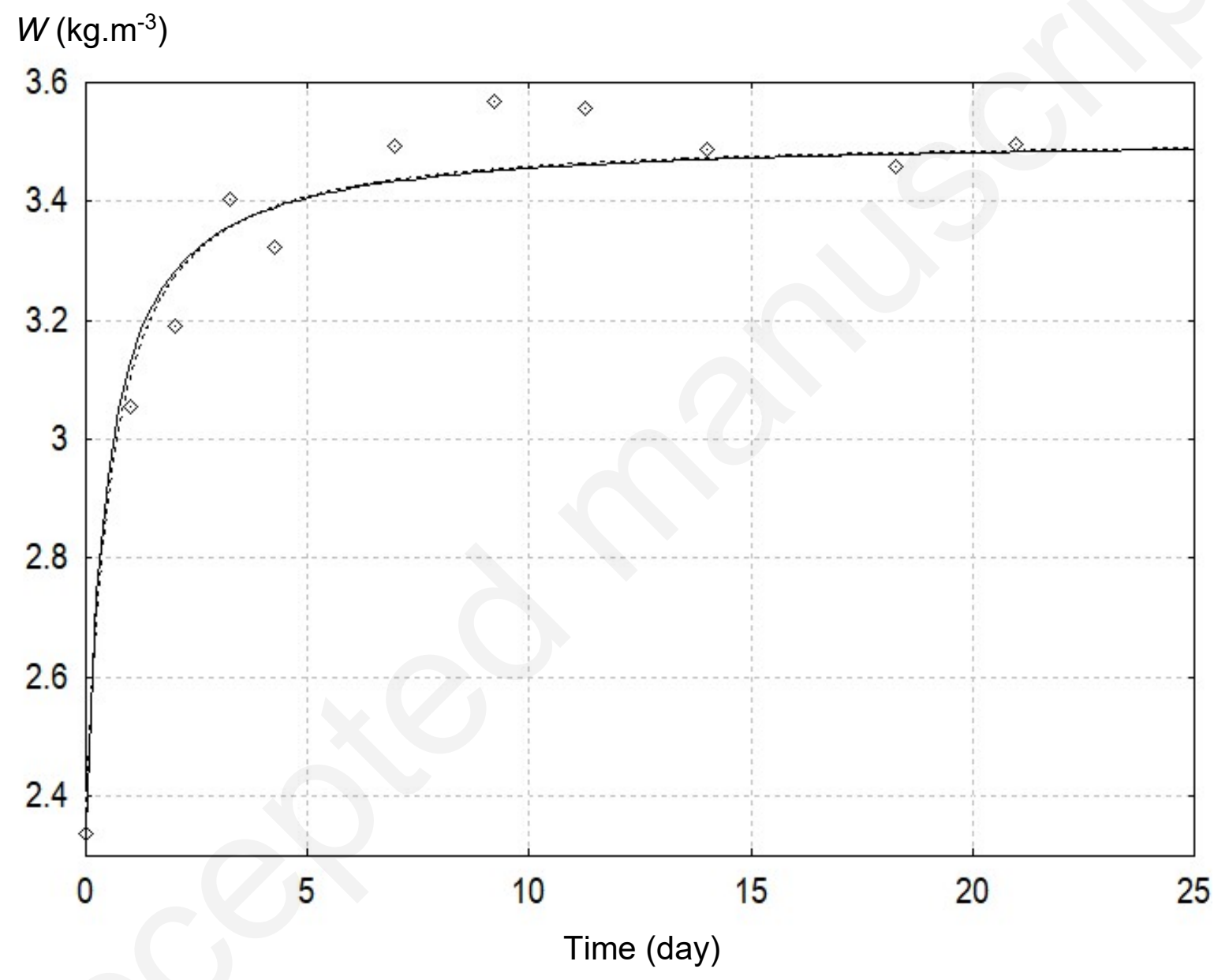

Fig. 9 